\title{
Molecular and physiological analysis of drought stress responses in Zea mays treated with plant growth promoting rhizobacteria
}

\author{
I. AHMAD ${ }^{1,5 *}$, S. ZAIB ${ }^{1}$, P.C.M.S. ALVES 2 , D.S. LUTHE ${ }^{3}$, A. BANO ${ }^{4}$, and S.N. SHAKEEL ${ }^{1 *}$ \\ Department of Biochemistry, Faculty of Biological Sciences, Quaid-i-Azam University, 45320, Islamabad, \\ Pakistan ${ }^{1}$ \\ Department of Plant Biotechnology, Universidade Federal de Lavras, 37200-00 Lavras, Brazil ${ }^{2}$ \\ College of Agricultural Sciences, Pennsylvania State University, University Park, 16802, PA, USA ${ }^{3}$ \\ Department of Plant Sciences, Faculty of Biological Sciences, Quaid-i-Azam University, 45320, Islamabad, \\ Pakistan ${ }^{4}$ \\ Department of Entomology, Pennsylvania State University, University Park, 16802, PA, USA
}

\begin{abstract}
Our research intended to appraise the performance of two different Pseudomonas strains on Zea mays L. (cv. B73) under drought stress and non-stress conditions. Plants were inoculated with P. putida KT2440 (Pp) and P. fluorescens (Pf1) followed by sampling at $0,3^{\text {rd }}$, and $6^{\text {th }}$ day after imposition of drought stress (DAS). Both strains demonstrated significant improvement in root length, protein content, chlorophyll content, and root and shoot fresh masses as compared to uninoculated drought stressed plants. Real-time quantitative PCR analysis revealed that drought stress responsive genes, i.e., the cold-related dehydrin 410 gene, WRKY18, and major facilitator superfamily were significantly down-regulated by Pf1 and Pp inoculation under drought stress condition on 6 DAS. Similarly, the down-regulated transcript abundance of lipoxygenase genes in inoculated plants on 6 DAS showed the role of Pf1 and Pp in scavenging reactive oxygen species under drought stress conditions. Among the selected jasmonic acid pathway responsive genes, maize protease inhibitor and 12-oxo-phytodienoatereductase 7 (OPR7) also revealed a potential role of these rhizobacteria under drought stress conditions. Seed inoculation of both strains significantly down-regulated the expression of $O P R 7$ gene under stress conditions. Our results advocate the complex growth promotion effects of both selected rhizobacterial strains and amelioration of the drought by modulating the expression of drought stress responsive genes.
\end{abstract}

Additional key words: chlorophyll, gene expression, maize, protein, Pseudomonas fluorescens, Pseudomonas putida.

\section{Introduction}

Plants are affected by various stressful environmental conditions and drought stress is one of the most destructive abiotic stresses in arid or semiarid regions. Global warming has also accelerated the harshness and frequency of drought and consequent reduction of food production (Vinocur and Altman 2005). The crop production and food security is also facing serious problem due to salinity and drought stresses (Munns and Tester 2008). Drought stress usually affects various biochemical and physiological processes like respiration, photosynthesis, ion uptake, nutrient metabolism, and translocation and hence reduces plant growth (Farooq et al. 2008, Jaleel et al. 2008). Drought stress response in plants is a very complex trait and it depends upon several factors such as genotype, severity of the stress, stress duration, environment, and developmental stage. The lack of water in the substrate

\footnotetext{
Submitted 2 February 2018, last revision 9 March 2019, accepted 19 March 2019.

Abbreviations: ABA - abscisic acid; CFU - colony forming units; COR - cold responsive; DAS - days after drought stress; Dhn1 - dehydrogenase1; JA - jasmonic acid; LOX - lipoxygenase; MFS - major facilitator super; MPI - maize protease inhibitor; OPR7 12-oxo-phytodienoate reductase 7; Pfi - Pseudomonas fluorescens; PGPR - plants growth promoting rhizobacteria; Pp - Pseudomonas putida; ROS - reactive oxygen species; RWC - relative water content.

Acknowledgements: This research was funded by the International Research Support Initiative Program (IRSIP), the Higher Education Commission (HEC), Pakistan, and the Quaid-i-Azam University Research Grant to Dr. Imtiaz Ahmad and Miss Sania Zaib. We would like to express our gratitude to all lab fellows especially Dr. Swayamjit Ray for his timely provision of all the lab facilities at Dr. Luthe's lab, Pennsylvania State University, PA, USA. We are also thankful to Dr. Gary Felton for providing all lab facilities at Department of Entomology, Pennsylvania State University, USA. We are also very thankful to two anonymous reviewers for their very helpful comments. The first two authors equally contributed to this work.

* Corresponding authors; e-mails: snq28@yahoo.com, imt849@gmail.com
} 
causes limited nutrient acquisition leading to indirect detrimental effects on plant growth (Benabdellah et al. 2011). Another indirect effect is production of reactive oxygen species (ROS), which reduce plant growth by lipid peroxidation, protein denaturation, and DNA mutation. For survival and resilience, plants evolve defensive strategies in response to certain stresses (Bohnert et al. 2006) resulting in production of several detoxifying and defensive enzymes, and compatible solutes (Molinari et al. 2007, Ray et al. 2016). The antioxidant enzymes scavenge free radicals and help in stress compensation and growth promotion (Zakikhani et al. 2012, Dourado et al. 2013, Nogueirol et al. 2015).

In order to ameliorate drought stress effects, researchers utilize different genomics and transgenic strategies in plants (Thudi et al. 2014). Modern biotechnological strategies have played a vital role in improving the drought stress tolerance in plants (Lu et al. 2013). With the advantage of plant breeding and genetic engineering, development of drought tolerant cultivars is also an effective approach to increase the crop yield and water use efficiency but genetically modified plants as a whole could not find a good place (Wahid et al. 2007). Symbiotic interactions of microbes with plants can help them survive a variety of biotic and abiotic stresses. Beneficial plant-microbe interactions can mitigate the stress effects in plants significantly (Marulanda et al. 2006). Stressed plants are dependent on plant growth promoting rhizobacteria (PGPR) that help in enhancing their metabolic activities to withstand the stress (Kavamura et al. 2013). The plant inoculation with such beneficial microbial communities helps in the growth promotion, stress tolerance, survival, and adaptation to changing climate conditions (Liddycoat et al. 2009, Marulanda et al. 2009, Benabdellah et al. 2011). Under drought stress, abscisic acid (ABA) biosynthesis increases and its catabolism is decreased eliciting the signals for closure of stomata in order to minimize the water loss and enhance the root network for maximizing the nutrients uptake (Bray 2002, De Smet et al. 2006), whereas ethylene production rises. Such effects of PGPR have also been attributed to a reduction of the ethylene production to compensate the normal ABA level in plants and ultimately protect them against damaging effects of drought stress (Arzanesh et al. 2011). Many previous studies have also shown the involvement of jasmonates in drought stress. Application of jasmonates, i.e., jasmonic acid (JA) or methyl jasmonate ameliorates water stress by increasing the antioxidative capacity of plants (Bandurska et al. 2003). Drought stress affects several genes and their products in plants at transcriptional, post-transcriptional, and translational levels. However, the knowledge of molecular mechanisms elucidating plantPGPR interactions in rhizosphere is scarce and needs attention (Nautiyal et al. 2013). Therefore, we used two different Pseudomonas strains (Pseudomonas putida KT2440 and Pseudomonas fluorescens Pf1) to study their effects on Zea mays L. (cv. B73) growth under drought and non-stress conditions. We studied the gene expression of different drought stress related, defense related, and JA related genes to understand underlying biochemical and physiological changes in the host plant elicited by selected PGPR. This study can boost the practical applicability of such biotechnological strategies especially in upcoming climate change.

\section{Materials and methods}

Bacterial inoculation: Both Pseudomonas fluorescens Pf1 and $P$. putida KT2440 strains obtained from the Department of Plant Sciences, Pennsylvania State University, PA, USA were grown on Luria-Broth (LB) medium at a temperature of $30 \pm 1{ }^{\circ} \mathrm{C}$ on an incubator shaker $(150 \mathrm{rpm})$ for $36 \mathrm{~h}$. The cultures were then spun (at $6000 \mathrm{~g}$ and $4^{\circ} \mathrm{C}$ for $10 \mathrm{~min}$ ). Pellets were washed thrice and absorbance of the culture was set at 1 at $600 \mathrm{~nm}=10^{7}-10^{8}$ colony forming units (CFU) $\mathrm{cm}^{-3}$ (Bhuvaneswari et al. 1980).

Plant growth and drought stress conditions: A pot experiment was conducted in order to compare the efficacy of two PGPR strains for maize growth improvement under drought stress condition. Maize Zea mays L. (cv. B73) seeds obtained from the Department of Plant Sciences, the Pennsylvania State University, PA, USA, were surface sterilized by soaking them in $1 \%(\mathrm{~m} / \mathrm{v})$ sodium hypochlorite for $3 \mathrm{~min}$ and then in $70 \%(\mathrm{v} / \mathrm{v})$ ethanol for $1 \mathrm{~min}$. The seeds were rinsed in distilled autoclaved water for three times and were incubated in bacterial suspension (P. putida KT2440 and P. fluorescens Pf1) or in distilled water (control) for $2 \mathrm{~h}$. The seeds were then sown in cylindrical shaped pots and after one week of germination, the seedlings were reduced to one per pot. Each pot contained $\sim 2 \mathrm{~kg}$ of the sterilized growth media containing a 1:1 mixture of soil (Hagerstown silt loam) and potting mix (commercial plant growth substrate, organic vigoro, Home Depot, USA). in a greenhouse, the average day/night temperatures were $22-28 / 14-17^{\circ} \mathrm{C}$, a 16-h photoperiod, an irradiance of $250 \mu \mathrm{mol} \mathrm{m} \mathrm{m}^{-2} \mathrm{~s}^{-1}$, and an air humidity of $60 \%$. Pots were arranged randomly with five biological replicates of each treatment. In order to maintain optimal soil moisture, almost equal amount of water was given to each pot for $21 \mathrm{~d}$ followed by drought stress for one week. The plants were used for all in situ measurements as growth parameters followed by sampling on $0,3^{\text {rd }}$, and $6^{\text {th }}$ day after drought stress imposition (DAS).

Assessment of growth parameters: Maize leaf samples were randomly collected on 0,3 , and 6 DAS with five biological replicates for each treatment group. Plant height was measured from the base of the plant to the tip of longest leaf. Root and shoot fresh masses and root length were measured from respective plants and the samples were dried at $60{ }^{\circ} \mathrm{C}$ for $72 \mathrm{~h}$ to determine dry masses. Samples for RNA extraction, cDNA synthesis, and PCR were immediately frozen at $-80{ }^{\circ} \mathrm{C}$. A chlorophyll meter (SPAD 502 Plus, Minolta, Tokyo, Japan) was used to assess the chlorophyll content of the leaf tissue (Markwell 1999). Third or fourth leaf was used for total protein content measurement by method of Lowry et al. (1951) using bovine serum albumin as a standard. 
In the case of relative water content (RWC) measurements, the $3^{\text {rd }}$ or $4^{\text {th }}$ leaf of maize plants was selected. After excision, the leaf was weighed (FW) and soaked in distilled water at $4{ }^{\circ} \mathrm{C}$ in the dark for $24 \mathrm{~h}$ to measure its fresh mass. The leaf was carefully dried with tissue paper and its turgid weight (TW) was measured. Then, it was oven dried at $72{ }^{\circ} \mathrm{C}$ for $48 \mathrm{~h}$ to measure dry weight. The RWC was calculated as previously described (Teulat et al. 2003) using a formula $\mathrm{RWC}[\%]=[(\mathrm{FW}-\mathrm{DW}) /(\mathrm{TW}-\mathrm{DW})] \times 100$.

Real-time quantitative PCR: Ribonucleic acic was extracted from three biological replicates for each day and treatment group by grinding leaf samples in liquid nitrogen using GenoGrinder 2000. TRIzol (Invitrogen, Carlsbad, USA) was used as an extraction reagent as recommended by the manufacturer ( $1 \mathrm{~cm}^{3}$ TRIzol for $0.1 \mathrm{~g}$ leaves). Quantification of RNA was done using a Nanodrop ND 2000 spectrophotometer (Thermo Fisher Scientific, San Jose, USA). Genomic DNA was digested by DNase treatment of the extracted RNA (Biolabs, New England, USA). Total extracted RNA (1 ug) was used as a template for the synthesis of cDNA with the help of high capacity
cDNA reverse transcription kit (Applied Biosystems, Foster City, USA) as recommended. Fast start universal SYBR Green Master Mix (Roche Applied Science, IN, USA) was used for the real-time PCR using a 7500 Fast real-time PCR system (Applied Biosystems). The PCR was conducted by using the conditions: $50^{\circ} \mathrm{C}$ for $2 \mathrm{~min}$ and $95^{\circ} \mathrm{C}$ for $10 \mathrm{~min}$; $95{ }^{\circ} \mathrm{C}$ for $30 \mathrm{~s}$ and $60^{\circ} \mathrm{C}$ for 1 min repeated in 35 cycles; $72{ }^{\circ} \mathrm{C}$ for $10 \mathrm{~min}$; and finally a dissociation stage. Sequences of all primers used in this study are listed in Table 1 Suppl. ZmActin was used as a reference gene because it is well known for the normalization (Stürzenbaum and Kille 2001). Transcript abundance was calculated by $\Delta \mathrm{Ct}=\mathrm{Ct}_{\text {target }}-\mathrm{Ct}_{\text {reference }}$ and values were expressed as $2^{-\Delta \Delta \mathrm{CT}}$. Fold changes in transcriptions were analyzed and compared between inoculated and control plants for each harvest time and treatment group.

Statistical analysis: For all the molecular and physiological parameters, we used two way ANOVA by using JMP Pro 13 (SAS Institute Inc, Cary, NC, USA) unless otherwise mentioned to test the effect of drought stress, PGPR inoculation, and correlation between drought stress and

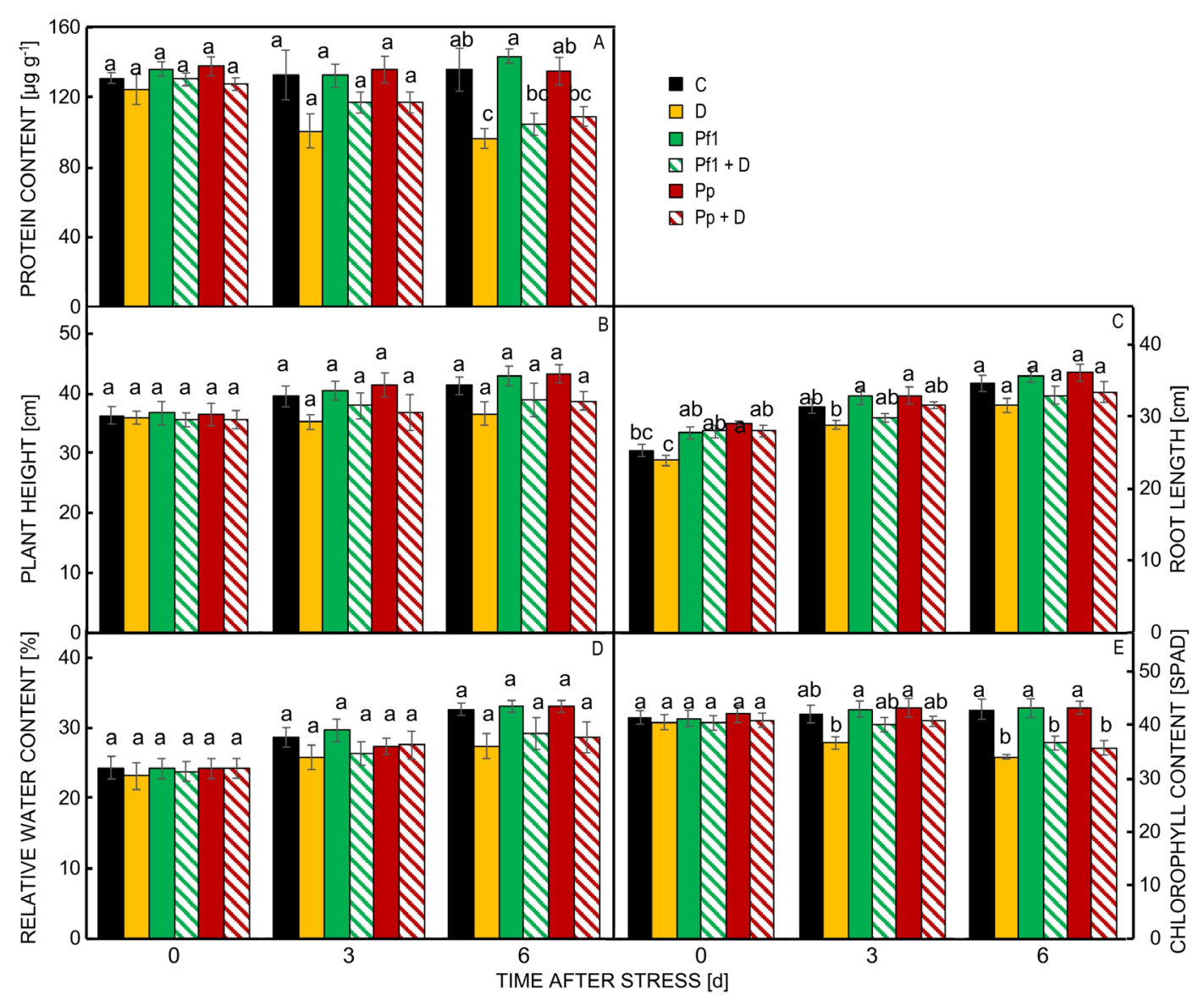

Fig. 1. Effects of plant growth promoting rhizobacteria (PGPR) on total protein content $(A)$, plant height $(B)$, root length $(C)$, relative water content $(D)$, and chlorophyll content $(E)$ of control maize plants $(C)$ or under drought stress (D). Plants were inoculated with Pseudomonas fluorescens (Pf1) or P. putida (Pp). Means $\pm \mathrm{SEs}, n=5$. Bars sharing common letters are not statistically different from each other at a $95 \%$ confidence level.romoting rhizobacteria (PGPR) on root fresh mass $(A)$, shoot fresh mass $(B)$, root dry mass $(C)$, and shoot dry mass $(D)$ of control maize plants $(C)$ or under drought stress (D). Plants were inoculated with Pseudomonas fluorescens (Pf1) or $P$. putida (Pp). Means \pm SEs, $n=5$. Bars sharing common letters are not statistically different from each other at a $95 \%$ confidence level. 
PGPR inoculation at $P<0.05$ for each sampling time. We used the Tukey HSD test for multiple comparisons of least squares means to test the effect of PGPR inoculation in the presence and absence of drought stress at each specific time point for all treatments.

\section{Results}

Different parameters including plant height, root length, root and shoot fresh and dry masses, relative water content, protein content, and chlorophyll content were measured on 0,3 and 6 DAS for evaluation PGPR effects.

There were no significant differences in plant height among different treatments. Our study revealed only some tendency that on 3 and 6 DAS, both Pfl and Pp strains helped plants to increase their height under stress and nostress conditions as compared to non-inoculated control plants (Fig. 1B). On 0 DAS, Pp inoculation more increased root length as compared to Pf1 inoculation and control. Under drought stress both strains significantly increased root length as compared to drought alone. On 3 DAS drought stress showed significant effects on root length whereas PGPR inoculation and their interactions were not statistically significant. Drought stress significantly reduced root length in all variants as compared to control. There was no significant difference observed for root length among different treatments for 6 DAS. Our data revealed that on 3 DAS, both Pfl and Pp strains inoculation helped plants to ameliorate drought stress by increasing the root length as compared to non-treated control plants (Fig. 1C).

As concerns RWC, no statistically significant differences were observed among different treatments for 0,3 , and 6 DAS. Our data shows that on 3 and 6 DAS, Pf1 and Pp strains slightly improved RWC as compared to nontreated control plants, but the results are not statistically significant (Fig. 1D).

Drought stress significantly decreased protein content on 6 DAS. The effects of PGPR inoculation and the interaction between drought stress and PGPR inoculation were not found statistically significant. Both Pfl and Pp were found to be effective for ameliorating the effect of drought stress on the protein content on 6 DAS as compared to non-inoculated control plants (Fig. 1A).

Drought stress significantly decreased chlorophyll content on 3 and 6 DAS. The PGPR inoculation and the interaction between drought stress and PGPR inoculation were insignificant. No significant differences among

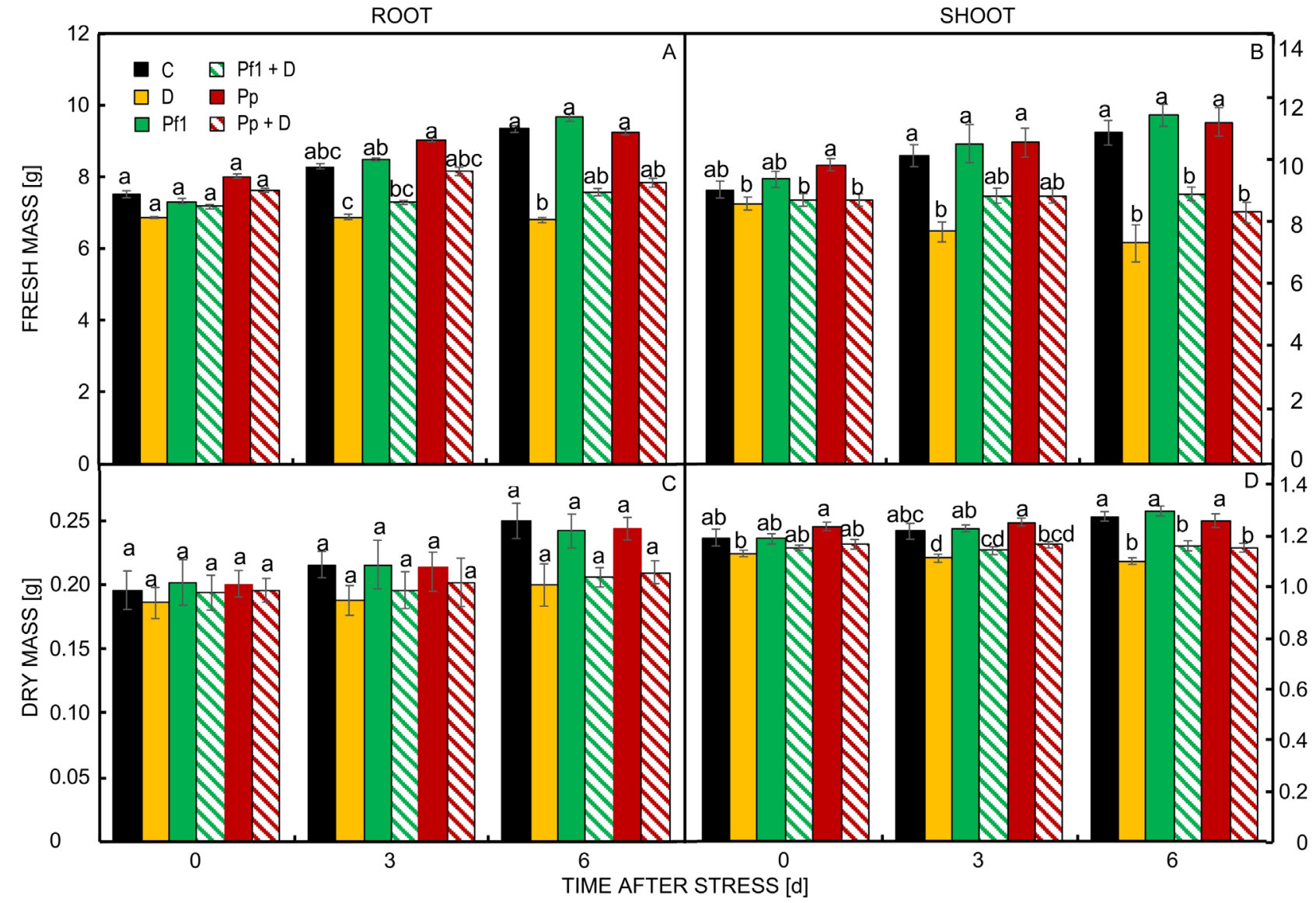

Fig. 2. Effects of plant growth promoting rhizobacteria (PGPR) on root fresh mass $(A)$, shoot fresh mass $(B)$, root dry mass $(C)$, and shoot dry mass $(D)$ of control maize plants $(C)$ or under drought stress (D). Plants were inoculated with Pseudomonas fluorescens (Pf1) or P. putida $(\mathrm{Pp})$. Means $\pm \mathrm{SEs}, n=5$. Bars sharing common letters are not statistically different from each other at a $95 \%$ confidence level. 
treatments were observed on 0 DAS as expected. More importantly, both Pf1 and Pp inoculations were found to be effective in the significant amelioration of drought stress and also under control conditions on 3 DAS as compared to non-inoculated control plants (Fig. 1E).

In order to ensure the positive effect of PGPR colonization in maize roots under drought stress and nonstress conditions, we assessed the root and shoot fresh and dry masses. For fresh root mass, no significant differences were observed among different treatments on 0 DAS. On 3 DAS, the drought stress and PGPR inoculation showed significant effects, whereas the interaction between drought stress and PGPR inoculation were not found significant. The Pp inoculation increased the root fresh mass as compared to Pf1 and control on 3 DAS. The root fresh mass under drought stress was also higher after Pp inoculation than under Pf1 inoculation and drought alone. On 6 DAS, a significant decrease in the root fresh mass of drought stressed plants was observed as compared to non-stress plants. Our study shows that both Pf1 and Pp inoculations were useful for ameliorating stress by increasing the root fresh mass (Fig. 2A).

In the case of shoot fresh mass, we noticed significant negative effects of drought stress on 0 and 3 DAS, whereas the effect of PGPR inoculation and their interactions were not significant. On 6 DAS, drought stress and PGPR inoculation showed significant effects, while their interactive effect was not significant. According to our observations, both Pf1 and Pp helped in mitigating the drought stress effect on 6 DAS (Fig. 2B).

For root dry mass no significant effects of drought stress, PGPR inoculation, and their interaction were observed among different treatments on 0,3 , and 6 DAS (Fig. 2C). For shoot dry mass, we observed significant effects of drought stress, whereas PGPR inoculation and the interactions between drought stress and PGPR inoculations were not statistically significant. Shoot dry mass of drought stressed plants was not different from other treatments on 0,3 , and 6 DAS. Shoot dry mass of drought stressed plants was significantly lower than of $\mathrm{Pp}$ inoculated plants on 0,3 , and 6 DAS and lower than of non-stressed non-inoculated control plants and nonstressed PGPR inoculated plants on 3 and 6 DAS. There was no difference in shoot dry mass of stressed inoculated plants and stress non-inoculated plants on 0,3 , and 6 DAS (Fig. 2D).

In order to check the effect of PGPR inoculation on drought responsive gene expression under drought stress,

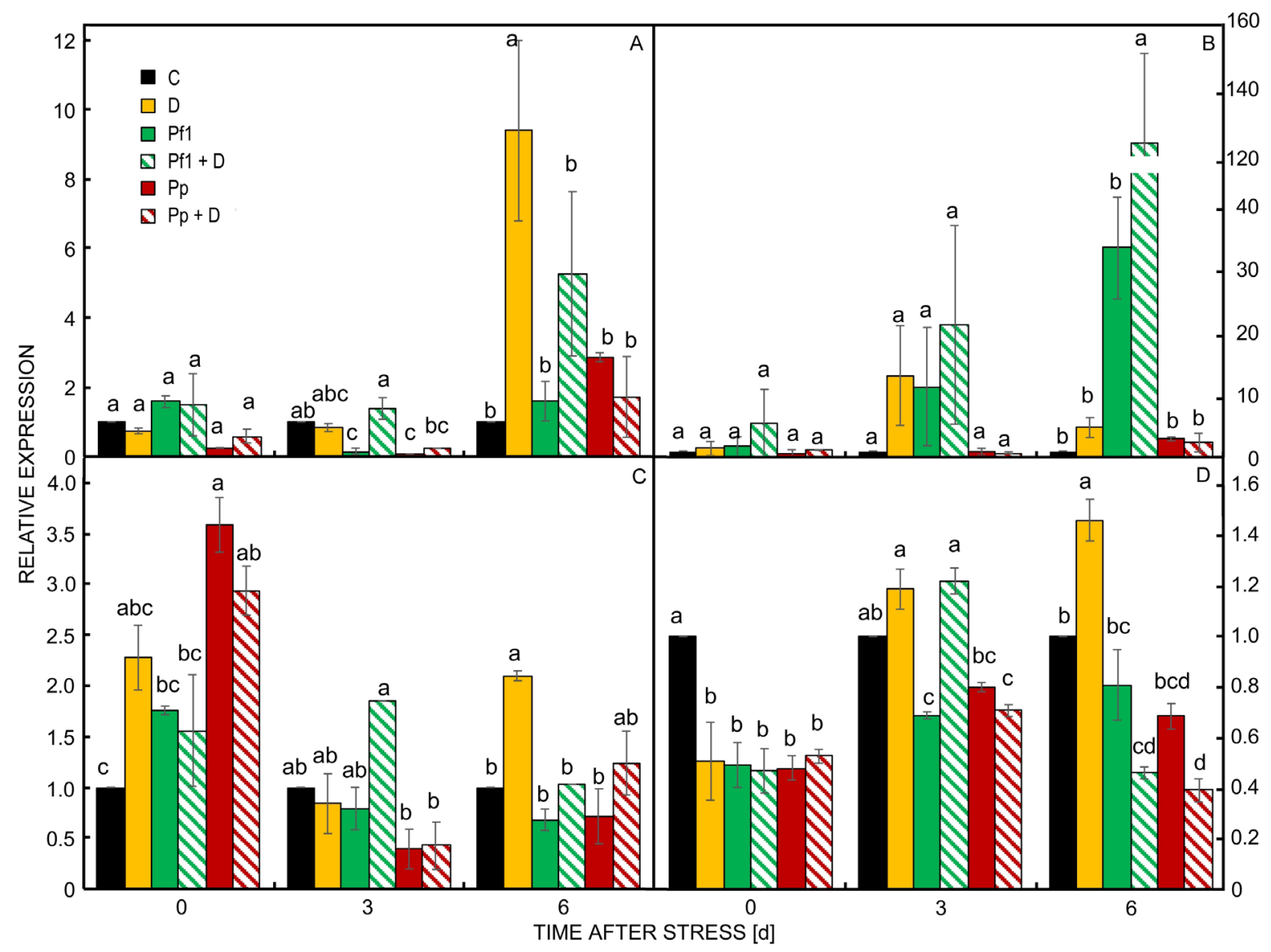

Fig. 3. Effects of plant growth promoting rhizobacteria (PGPR) on selected drought stress responsive genes COR410 (A), DHN1 $(B)$, WRKY18 (C), and MFS (D) of control maize plants (C) or under drought stress (D). Plants were inoculated with Pseudomonas fluorescens (Pf1) or P. putida (Pp). Means \pm SEs, $n=5$. Bars sharing common letters are not statistically different from each other at a $95 \%$ confidence level. 
we assessed the relative transcript abundance of some important genes in PGPR inoculated and non-inoculated maize plants under stressed versus non-stressed conditions. Significant up-regulation of $Z$. mays COR410 gene (a dehydrin gene family member associated with freezing tolerance) expression was found in plants under prolonged drought stress (6 DAS). Interestingly, Pf1 inoculation repressed the COR410 expression 2 - 3 folds, whereas Pp inoculation by 3 - 4 folds on 6 DAS. No significant difference of COR410 expression was observed among different treatments on 0 DAS (Fig. $3 A$ ).

The Dhnl (dehydrogenase1) gene also belongs to the dehydrin family that responds to water deficiency in several plants. No significant differences in its expression was observed among different treatments on 0 and 3 DAS. At 6 DAS, effects of drought stress, PGPR inoculation, and their interactions were found statistically significant. The Pf1 inoculation induced a gradual up-regulation of Dhnl transcription on 3 and 6 DAS and no significant differences in the $\mathrm{Pp}$ inoculated plants was observed in all the treatments and time points. Our results show that Dhnl expression is induced in maize under drought stress, and $P$. fluorescens inoculation helped in relative enhanced expression during prolonged stress at 6 DAS (Fig. $3 B$ ).

The $W R K Y$, a major transcription factors family of plants, has a regulatory function (Eulgem and Somssich 2007) in several plant processes including coping with biotic and abiotic stresses. In response to several stress stimuli, the plants start recruiting various WRKYs for downstream cascade regulations. We selected WRKY18 gene to study its involvement in drought stress response and interactive role in case of selected PGPR. For WRKY18 gene on 0 DAS, the model predicted significant effects of PGPR inoculation and the interaction between drought stress and PGPR inoculation, whereas drought stress did not show any significant difference. Significant up-regulation of WRKY18 transcripts was observed when treated with Pp as compared to Pf1 on 0 DAS. Similarly, for 3 DAS, significant effects of PGPR inoculation and their interaction were observed, while effect of drought stress was not significant. On 6 DAS, significant effects of drought stress and PGPR inoculation were observed, whereas their interaction was not found to be statistically significant. There was significant up-regulation of WRKY18 transcription in drought-stressed plants as compared to other treatments on 6 DAS. Interestingly, our data show the evidence of a reduced basal level of WRKY18 transcription in the presence of drought stress in comparison to control samples. This might be due to less availability of motifs or domains required for the binding of WRKY TFs under our

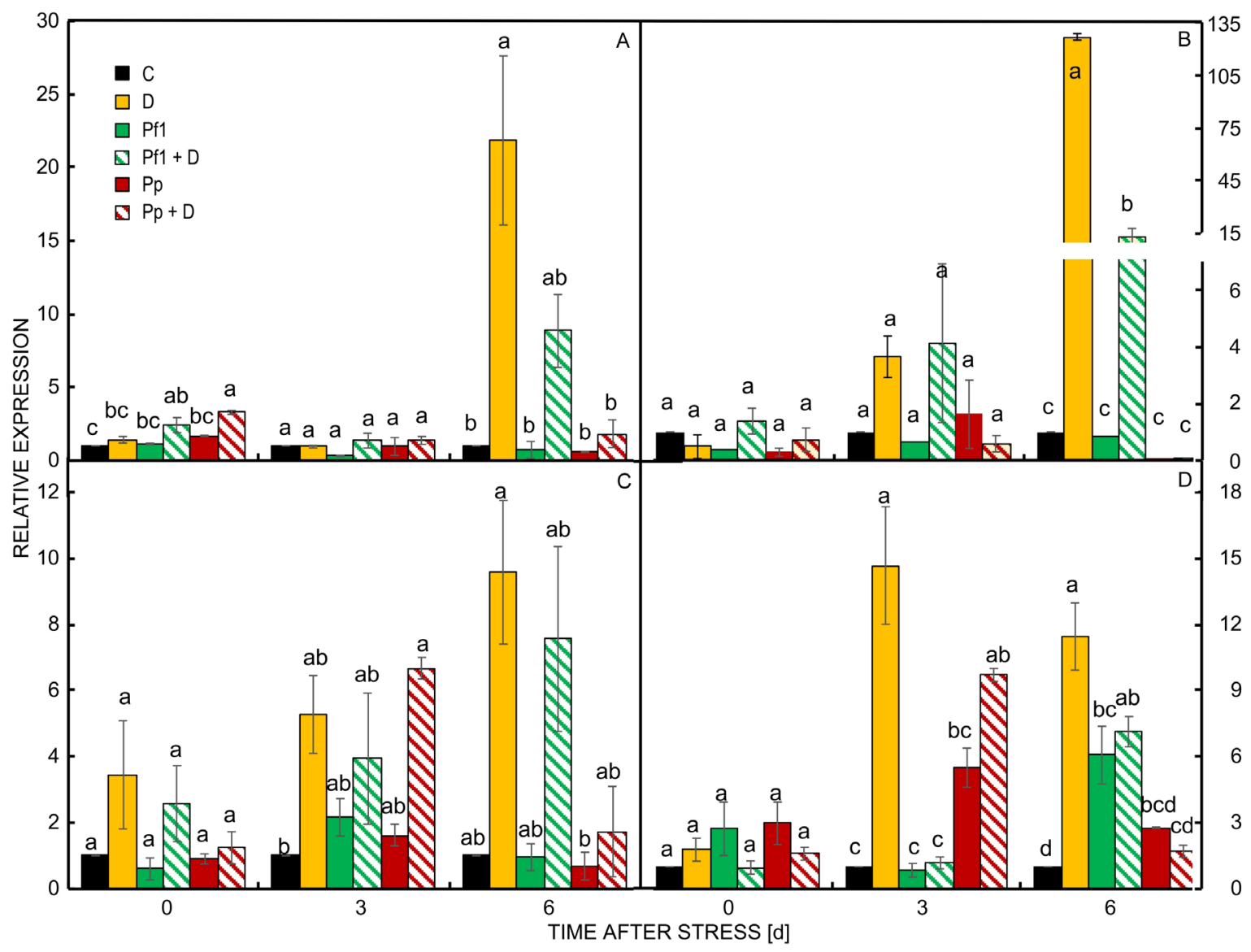

Fig. 4. Effects of plant growth promoting rhizobacteria (PGPR) on selected lipogenase pathway genes LOX1 (A), LOX3 (B), LOX5 (C), and LOX6 (D) of control maize plants (C) or under drought stress (D). Plants were inoculated with Pseudomonas fluorescens (Pf1) or $P$. putida $(\mathrm{Pp})$. Means \pm SEs, $n=5$. Bars sharing common letters are not statistically different from each other at a $95 \%$ confidence level. 
experimental conditions (Fig. 3C).

The major facilitator super (MFS) family, a group of secondary transporters, was significantly affected by drought stress, PGPR inoculation, and their interactions already on 0 DAS. Similarly, we observed significant effects of these treatments on 3 DAS, whereas on 6 DAS, PGPR inoculation and the interaction between drought stress and PGPR inoculation were found statistically significant, but the effect of drought stress was not significant. Interestingly, both Pf1 and Pp strains seem to be effective in controling the membrane transport under prolonged stress (6 DAS) as evident by reduced $M F S$ transcription in inoculated plants under drought stress (Fig. 3D).

The ZmLOX1, ZmLOX3, ZmLOX5, and ZmLOX6 belong to lipoxygenases (LOXs) that respond to dehydration stress in plants (Lyons et al. 2013). Significant up-regulation of $L O X 1$ transcription was observed in plants under drought treatment as compared to control on 6 DAS only. Though small up-regulation of LOX1 genes expression was found after PGPR inoculation on 0 DAS, no significant effects of treatments were found on 3 DAS (Fig. 4A). Similarly, significant up-regulation of $L O X 3$ expression was observed in drought-stressed plants as compared to control on 6 DAS. This upregulation was higher than that in drought-stressed inoculated plants. No significant differences were observed in expression of $L O X 3$ gene among different treatments on 0 and 3 DAS (Fig. 4B). No significant differences in LOX5 and LOX6 expression among different treatments were observed on 0 DAS (Fig. 4C,D). Drought stress alone or in combination with inoculation significantly increased $L O X 5$ expression on 3 and 6 DAS (Fig. 4C). The LOX6 gene responded by up-regulating significantly in drought stressed noninoculated maize plants upto 14-folds on 3 DAS followed by reduction on 6 DAS. These up-regulations were higher than those in drought-stressed inoculated plants (Fig. 4D). Our data show that, as compared to non-inoculated drought stressed plants, PGPR inoculation might have had a stress amelioration effect on the maize plants in order to withstand the severity of the stress, but this effect was variable for different growth parameters, which may be dependent on the strain used, plant variety, and severity and duration of stress.

Jasmonic acid pathway has a potential role in drought stress through some known and some unexplored cross talks. In order to explore this, the changes in transcription of some JA pathway genes were also assessed. Transcription of a maize protease inhibitor $(M P I)$ showed an insignificant difference among different treatments on 0 and 3 DAS. On

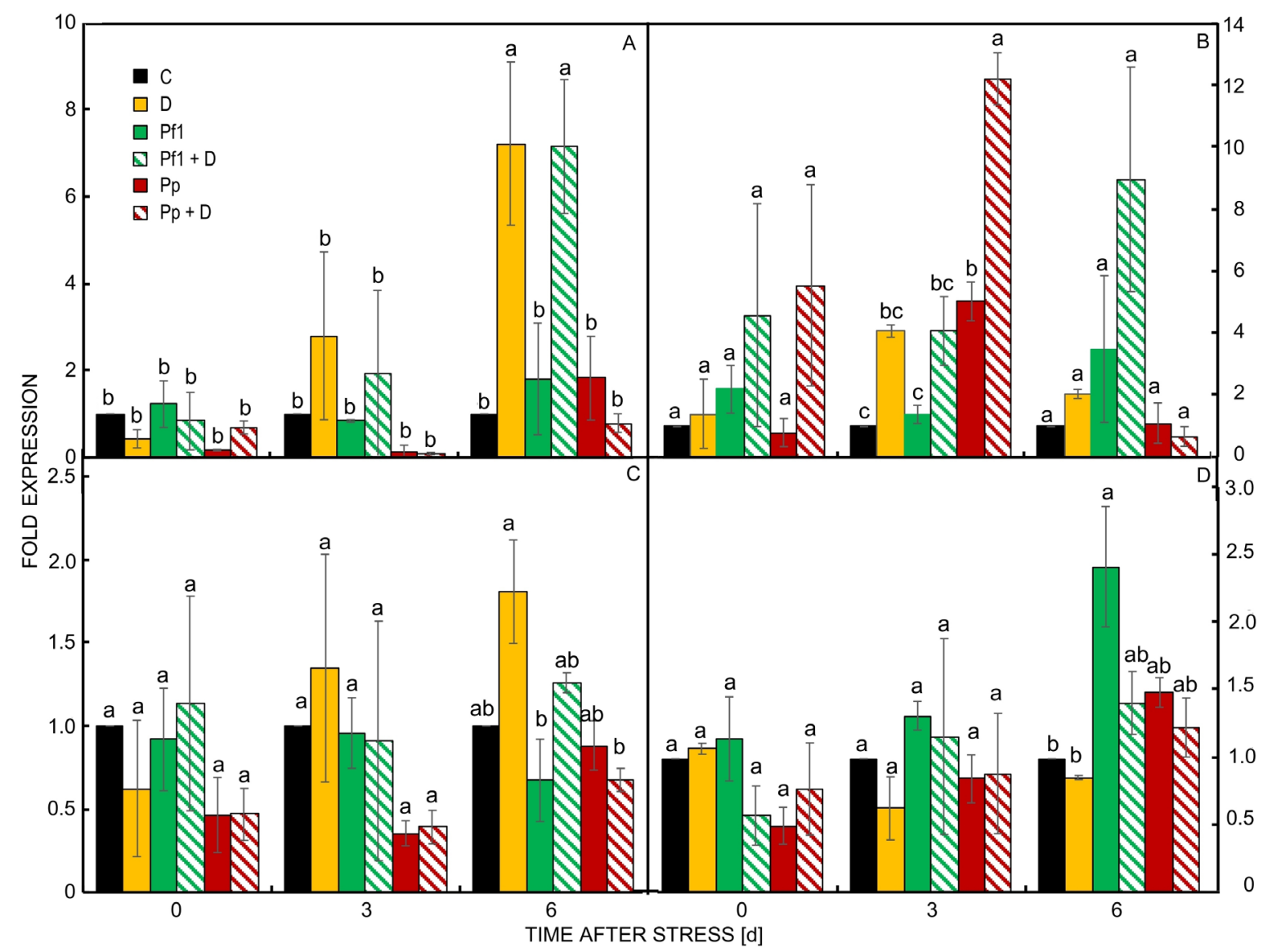

Fig. 5. Effects of plant growth promoting rhizobacteria (PGPR) on selected jasmonic acid pathway genes MPI $(A), R I P 2(B), O P R 7(C)$, and HPL $(D)$ of control maize plants $(\mathrm{C})$ or under drought stress (D). Plants were inoculated with Pseudomonas fluorescens (Pf1) or P. putida $(\mathrm{Pp})$. Means $\pm \mathrm{SEs}, n=5$. Bars sharing common letters are not statistically different from each other at a $95 \%$ confidence level. 
6 DAS, the effects of drought stress and the interaction between drought stress and PGPR inoculation were significant, whereas the effect of PGPR inoculation was not significant. Our data suggests the positive role of PGPR in ameliorating the effect of drought stress (Fig. $5 \mathrm{~A}$ ). For ribosome inactivating protein 2 (RIP2) gene expression, no significant effects of drought stress, PGPR inoculation, and their interactions were observed on 0 and 6 DAS. However, on 3 DAS, there were significant effects of drought stress, PGPR inoculation, and the interaction between drought stress and PGPR inoculation. The highest RIP2 expression was observed in drought-stressed plants inoculated with Pp (Fig. 5B). Plant oxo-phytodienoate reductases $(O P R s)$ are usually expressed under different environmental stimuli inducing JA, but their biochemical or physiological roles are still unclear. No significant differences in expression of OPR7 were observed on 0 and 3 DAS. On 6 DAS, drought stress and PGPR inoculation showed significant effects, whereas the interaction between drought stress and PGPR inoculation was not found statistically significant. A higher up-regulation of $O P R 7$ transcription was observed in drought-stressed non-inoculated plants as compared to inoculated ones (Fig. 5C). Hydrogen peroxide lyase $(H P L)$ gene is thought to play a role in mediating plant defense responses. No significant effects of drought stress, PGPR inoculation and their interaction were observed in case of HPL transcript among different treatments on 0 and 3 DAS. Only PGPR inoculation showed a significant effect on 6 DAS: HPL expression in Pf1 inoculated treated plants was highest (Fig. 5D).

\section{Discussion}

The PGPR play important roles in plant growth, development, and physiology. They promote the growth of the plants by various mechanisms like production of siderophores, antibiotics, phytohormones, by phosphate solubilization in substrate, and by nitrogen fixation. They help plants to withstand different stress conditions by activation of defense pathways and downstream signaling. The Pp and Pfl strains are known as an important and largest group of rhizobacteria with plant growth promotion properties, potentially known as biocontrol (Kloepper et al. 1992). In current study, the maize treated with Pp and Pf1 under drought stress showed increased protein content, root length, chlorophyll content, fresh root and shoot fresh and dry masses relative to non-inoculated plants.

It has been reported that plants reduce the biosynthesis of certain proteins and increase the biosynthesis of defensive proteins to overcome drought stress trauma in plants, which is also dependent on the intensity and duration of stress. Despite the defensive proteins being produced in response to stress conditions, the total soluble protein content decreases gradually with intensity and duration of stress due to a decrease in the rate and efficiency of photosynthesis (Mohammadkhani and Heidari 2008). Another reason for a reduced protein content can be the accelerated rate of protein degradation due to enhanced protease activity and activities of other catabolic enzymes, which is characteristic for oxidative stress induced by drought stress. (Moran et al. 1994). Our study also exhibits similar results for protein in drought treated plant groups when compared with control groups. However, this effect was ameliorated significantly on $6 \mathrm{DAS}$ in the PGPR inoculated plant group under drought stress condition.

Plants under drought stress tend to decrease the water loss with the help of different defense strategies, whereas simultaneously increase the nutrient uptake by enhancing the root network. The occurrence of Pseudomonas sp. in and around the root system of cereals, vegetables and their beneficial effects upon inoculation has been well studied. In our study, a significant decrease in root length of drought treated plant groups was observed started on 3 DAS as compared to the control treatment. Both PGPR strains significantly ameliorated the drought stress by increasing the root length as compared to non-inoculated drought stressed control plants. The promotion in growth parameters might be due to the synthesis of bacterial growth hormones, e.g., indole acetic acid and gibberellins (Khalid et al. 2004) resulting in increased nutrient uptake in plants. Because of rapid colonization of the roots, these rhizobacteria are widely used as soil inoculants in many crops for growth promotion.

Drought stress can easily be judged by RWC (Fisher et al. 2000), as it is significantly reduced in leaves (Nayyar and Gupta 2006). Stressful conditions can be mitigated in the presence of PGPR due to their growth promoting effects on the physiology and molecular biology of the plants (Dodd et al. 2010). In this study, we did not observe any difference in RWC of both inoculated and non-inoculated maize plants exposed to the drought stress as compared to control plants.

The rate of photosynthesis in plants usually decreases under drought stress (Kawamitsu et al. 2000) because of reduced stomatal conductance to lower the water loss leading to the reduced $\mathrm{CO}_{2}$ fixation. Also the chlorophyll content and the photosynthetic machinery are affected under drought stress causing the inhibition of photosynthesis (Iturbe-Ormaetxe et al. 1998, Manivannan et al. 2007). Many studies on Triticum aestivum (Gajewska and Skłodowska 2007), Z. mays (Krantev et al. 2008), Brassica juncea (Alam et al. 2007), Bruguiera gymnorrhiza, and Kandelia candel (Huang and Wang 2010) have reported the decrease in chlorophyll content under stress conditions. Our results are consistent with previous studies indicating that the drought stress significantly reduced the chlorophyll content on 3 and 6 DAS. The PGPR were found to be helpful for the plants by increasing the chlorophyll content on 3 DAS when compared with non-inoculated plants under drought stress.

The drought stress also negatively affected the root and shoot fresh and dry masses, however, PGPR-treated plants had significantly higher root fresh mass on 3 and 6 DAS and shoot fresh and dry masses on 3 DAS when compared with the stressed non-inoculated plants. This might be because of their ability to help the plants in increasing their above-ground and below-ground growth by nutrients acquisition, assimilation, enhanced roots networking and 
metabolites production.

Complex multi-component signaling pathways are triggered in plants in order to restore cellular homeostasis and to promote survival under different abiotic stresses but the knowledge about these molecular mechanisms is still limited. Some drought stress inducible genes respond directly to the stress, whereas some genes respond after the accumulation of ABA. All these genes have protective roles against the stress (Bray 1997). Transcripts of such genes usually accumulate under drought stress conditions. A PGPR inoculation can reduce the severity of the stress by regulating the expression of defense related genes, which can play a role in counteracting the damage caused by dehydration conditions. In order to explore the role of PGPR, we analyzed several genes for their relative expression under given conditions using real-time qPCR.

The ZmCOR410 and Dhnl belong to the dehydrin gene family that are over-expressed under dehydration conditions in maize plants and due to ABA exposure (Danyluk et al. 1998). In our study, induced expression of Z. mays COR 410 under drought stress conditions in non-inoculated plants showed evidence of plant cell dehydration due to drought stress at 6 DAS. Induced expression of COR410 gene to several folds is interesting. Characterization of such regulatory genes in signaling pathways may help devise strategies to improve plant-rhizobacterial interactions. In our experiment, $P$. fluorescens $\mathrm{Pf1}$ and $P$. putida KT2440 inoculations may have reduced the dehydration damage by down-regulating COR410 expression in PGPR inoculated stressed and PGPR inoculated non-stressed plants as compared to non-inoculated stressed plants to help the plants to withstand stress. Similarly, Dhnl gene expression was up-regulated almost 13 -folds under drought stress in non-inoculated plants on 3 DAS but decreased almost 7-folds on 6 DAS showing turnover of Dhnl transcription after prolonged drought stress. The Pf1 inoculation induced a gradual up-regulation of $D h n 1$ gene on 0,3 , and 6 DAS, which was significant on 6 DAS. The Dhnl gene contribute in osmotic stress tolerance and Pf1 was found to be more efficient for inducing its expression as compared to $\mathrm{Pp}$.

The WRKY transcription factors target downstream genes that are important for detoxification and scavenging ROS like peroxidases, LOXs LOX1, and glutathione-Stransferases (Jiang and Deyholos 2009). Due to the role of WRKY18 in osmotic stress response, ROS scavenging activity becomes likely (Remy et al. 2013). Some changes in transcription of WRKY18 gene in the case of drought stress and inoculations were observed. Non-inoculated drought stressed plants showed up-regulation of WRKY18 gene expression on 0 and 6 DAS compared to PGPR inoculated drought stressed plants and non-inoculated non-stressed plants. The PGPR inoculation might have reduced the effect of drought stress on 6 DAS by lowering the expression of WRKY18 gene.

The MFS family is a group of secondary transporters required for the transport across the cellular membranes using proton gradient as an energy source (Petrasek et al. 2006). Recently, MFS has also been assigned a dual role in Arabidopsis for drought stress tolerance and polar auxin transport through regulating movements of stomata and auxin transport by changing proton and potassium transition (Remy et al. 2013). Our data show a significant up-regulation of $M F S$ gene expression on 3 and 6 DAS in non-inoculated maize plants under drought stress, which was significantly repressed by $\mathrm{Pp}$ inoculation on 3 and 6 DAS and by Pfl inoculation on 6 DAS. These data show that PGPR inoculation might have modulated the expression of these defense genes to help withstand the drought stress.

Current research emphasizes the central role of ROS production in cells as a signaling interface in plant adaptation to salt and drought stresses. The ROS in excess are scavenged particularly through antioxidant metabolites like glutathione, ascorbate, and tocopherols. etc., and through ROS detoxifying enzymes like catalase, superoxide dismutase, ascorbate peroxidase, etc. (Mittler 2002). Lipoxygenases are involved in various physiological processes (Brash 1999). They are nonheme iron containing dioxygenases, which catalyze the conversion of lipids and polyunsaturated fatty acids into hydroperoxy fatty acids, which are further degraded into oxylipins such as jasmonic acid, methyl jasmonic acid, and traumatin (Blée 2002). Oxylipins are known to play an important role in development, senescence, formation of flavor compounds, and defense responses to abiotic and biotic stresses (Williams et al. 2000, Ye et al. 2000, Vellosillo et al. 2007, Yang et al. 2012). We also assessed the expression pattern of the genes involved in LOX pathway, such as LOX1, LOX3, LOX5, and LOX6, to characterize the role of our selected PGPR strains in drought stress amelioration. These genes were up-regulated under drought stress possibly to scavenge ROS so as to overcome the stress. The $L O X 1, L O X 3, L O X 5$ genes were significantly up-regulated on 6 DAS in non-inoculated drought stressed maize plants, whereas LOX6 gene significant up-regulation started on 3 DAS and also showed a significant up-regulation on 6 DAS. Both Pseudomonas putida KT2440 and $P$. fluorescens Pf1 inoculations might have helped in withstanding drought stress through ROS scavenging on 6 DAS. P. putida KT2440 was found to be more efficient as compared to P. fluorescens Pf1 for repressing the expression of these genes.

A wide range of abiotic stresses can induce JA signaling like drought, wounding (Doares et al. 1995), osmotic stress (Kramell et al. 1995), and also the extracts of yeasts (Parchmann et al. 1997). Stress-inducible genes play a role both in initial stress responses and also in establishing stress tolerance in plants. Exogenous JA is found to protect the plants from drought induced stress through enhancing the activity of antioxidant enzymes (Nafie et al. 2011). Over-expression of JA pathway related genes in transgenic plants also improves osmotic stress tolerance in addition to showing ABA-hypersensitive phenotypes (Shinozaki and Yamaguchi-Shinozaki 2004). Moreover, drought stress also raises the endogenous JA content in maize root cells (Xin et al. 1997). Our results demonstrate the involvement of $M P I$, and $O P R 7$ in drought stress response as a defense mechanism leading to stress amelioration. The MPI expression showed a gradual increase in drought 
stressed plants on 0,3 , and 6 DAS, and it was downregulated in $\mathrm{Pp}$ inoculated drought stressed plants on 3 and 6 DAS, whereas in Pf1 inoculated drought stressed plants on 3 DAS only. The transcript abundance of OPR7 gene was higher on 6 DAS in non-inoculated drought stressed samples than in Pf1 inoculated non stressed plants and Pp inoculated drought stressed plants, which might have attributed tolerance to the plants against drought stress.

Many studies on plant tolerance to drought showed that PGPR inoculation improves drought tolerance by affecting the transcription of drought stress responsive genes (Sarma and Saikia 2014) and also the phytohomonal balance (Figueiredo et al. 2008). In our current study, drought stress significantly affected the protein and chlorophyll by lowering their content and also decreased root length and shoot and root biomasses. However, PGPR treatment under stress helped plants to improve their growth most probably through nutrient uptake. At the transcriptional level, PGPR mediated down regulation of many drought stress responsive genes, i.e., COR410, WRKY18, and MFS, and also of JA pathway responsive genes, $L O X, M P I$, and $O P R 7$, might have helped plants in tolerating the drought stress.

The results presented in this work suggest that the P. putida KT2440 and P. fluorescens Pf1 are efficacious PGPR strains against drought stress in maize plants possibly by attenuating the transcript levels of drought stress responsive genes, lipoxygenase pathway genes, and JA pathway responsive genes. Collectively, these responses can facilitate the maize plants from desiccation under stressful conditions.

\section{References}

Alam, M.M., Hayat, S., Ali, B., Ahmad, A.: Effect of 28-homobrassinolide treatment on nickel toxicity in Brassica juncea. - Photosynthetica 45: 139-142, 2007.

Arzanesh, M., Alikhani, H., Khavaz,i K., Rahimian H., Miransari M.: Wheat (Triticum aestivum L.) growth enhancement by Azospirillum sp. under drought stress. - World J Microbiol. Biotechnol. 27: 197-205, 2011.

Bandurska, H., Stroiński, A., Kubiś, J.: The effect of jasmonic acid on the accumulation of ABA, proline and spermidine and its influence on membrane injury under water deficit in two barley genotypes. - Acta Physiol. Plant. 25: 279-285, 2003.

Benabdellah, K., Abbas, Y., Abourouh, M., Aroca, R., Azcón, R.: Influence of two bacterial isolates from degraded and nondegraded soils and arbuscular mycorrhizae fungi isolated from semi-arid zone on the growth of Trifolium repens under drought conditions: mechanisms related to bacterial effectiveness. - Eur. J. Soil Biol. 47: 303-309, 2011.

Bhuvaneswari, T., Turgeon, B.G., Bauer, W.D.: Early events in the infection of soybean (Glycine max L. Merr) by Rhizobium japonicum I. Localization of infectible root cells. - Plant Physiol. 66: 1027-1031, 1980.

Blée, E.: Impact of phyto-oxylipins in plant defense. - Trends Plant Sci. 7: 315-322, 2002.

Bohnert, H.J., Gong, Q., Li, P., Ma, S.: Unraveling abiotic stress tolerance mechanisms - getting genomics going. - Curr. Opin. Plant Biol. 9: 180-188, 2006.

Brash, A.R.: Lipoxygenases: occurrence, functions, catalysis, and acquisition of substrate. - J. biol. Chem. 274: 23679-
23682, 1999.

Bray, E.: Abscisic acid regulation of gene expression during water-deficit stress in the era of the Arabidopsis genome. Plant Cell Environ. 25: 153-161, 2002.

Bray, E.A.: Plant responses to water deficit. - Trends Plant Sci. 2: 48-54, 1997.

Danyluk, J., Perron, A., Houde, M., Limin, A., Fowler, B., Benhamou, N., Sarhan, F.: Accumulation of an acidic dehydrin in the vicinity of the plasma membrane during cold acclimation of wheat. - Plant Cell 10: 623-638, 1998.

De Smet, I., Zhang ,H., Inzé, D., Beeckman, T.: A novel role for abscisic acid emerges from underground. - Trends Plant Sci. 11: 434-439, 2006.

Doares, S.H., Narváez-Vásquez, J., Conconi, A., Ryan, C.A.: Salicylic acid inhibits synthesis of proteinase inhibitors in tomato leaves induced by systemin and jasmonic acid. - Plant Physiol. 108: 1741-1746, 1995.

Dodd, A.N., Kudla, J., Sanders, D.: The language of calcium signaling. - Annu. Rev. Plant Biol. 61: 593-620, 2010.

Dourado, M., Martins, P., Quecine, M., Piotto, F., Souza, L., Franco, M., Tezotto, T., Azevedo, R.: Burkholderia sp. SCMS54 reduces cadmium toxicity and promotes growth in tomato. - Ann. appl. Biol. 163: 494-507, 2013.

Eulgem, T., Somssich, I.E.: Networks of WRKY transcription factors in defense signaling. - Curr. Opin. Plant Biol. 10: 366 371, 2007.

Farooq, M., Basra, S., Wahid, A., Cheema, Z., Cheema, M., Khaliq, A.: Physiological role of exogenously applied glycinebetaine to improve drought tolerance in fine grain aromatic rice (Oryza sativa L.). - J. Agron. Crop Sci. 194: 325-333, 2008.

Figueiredo, M.V., Burity, H.A., Martínez, C.R., Chanway, C.P.: Alleviation of drought stress in the common bean (Phaseolus vulgaris L.) by co-inoculation with Paenibacillus polymyxa and Rhizobium tropici. - Appl. Soil Ecol. 40: 182-188, 2008.

Fisher, D., Steiner, J., Endale, D., Stuedemann, J., Schomberg, H., Franzluebbers, A., Wilkinson, S.: The relationship of land use practices to surface water quality in the Upper Oconee Watershed of Georgia. - Forest Ecol. Manage. 128: 39-48, 2000.

Gajewska, E., Skłodowska, M.: Effect of nickel on ROS content and antioxidative enzyme activities in wheat leaves. BioMetals 20: 27-36, 2007.

Huang, G.-Y., Wang, Y.-S.: Physiological and biochemical responses in the leaves of two mangrove plant seedlings (Kandelia candel and Bruguiera gymnorrhiza) exposed to multiple heavy metals. - J. hazard. Mater. 182: 848-854, 2010.

Iturbe-Ormaetxe, I., Escuredo, P.R., Arrese-Igor, C., Becana, M.: Oxidative damage in pea plants exposed to water deficit or paraquat. - Plant Physiol. 116: 173-181, 1998.

Jaleel, C.A., Manivannan, P., Lakshmanan, G., Gomathinayagam, M., Panneerselvam, R.: Alterations in morphological parameters and photosynthetic pigment responses of Catharanthus roseus under soil water deficits. - Colloids Surf. B. Biointerfaces 61: 298-303, 2008.

Jiang, Y., Deyholos, M.K.: Functional characterization of Arabidopsis NaCl-inducible WRKY25 and WRKY33 transcription factors in abiotic stresses. - Plant mol. Biol. 69: 91-105, 2009.

Kavamura, V.N., Santos, S.N., Da Silva, J.L., Parma, M.M., Ávila, L.A., Visconti, A., Zucchi, T.D., Taketani, R.G., Andreote, F.D., De Melo, I.S.: Screening of Brazilian cacti rhizobacteria for plant growth promotion under drought. Microbiol. Res. 168: 183-191, 2013.

Kawamitsu, Y., Driscoll, T., Boyer, J.S.: Photosynthesis during desiccation in an intertidal alga and a land plant. - Plant Cell 
Physiol. 41: 344-353, 2000.

Khalid, A., Arshad, M., Zahir, Z.: Screening plant growth promoting rhizobacteria for improving growth and yield of wheat. - J. appl. Microbiol. 96: 473-480, 2004.

Kloepper, J.W., Tuzun, S., Kuć, J.A.: Proposed definitions related to induced disease resistance. - Biocontrol Sci. Technol. 2: 349-351, 1992

Kramell, R., Atzorn, R., Schneider, G., Miersch, O., Brückner, C., Schmidt, J., Sembdner, G., Parthier, B.: Occurrence and identification of jasmonic acid and its amino acid conjugates induced by osmotic stress in barley leaf tissue. - J. Plant Growth Regul. 14: 29-36, 1995.

Krantev, A., Yordanova, R., Janda, T., Szalai, G., Popova, L.: Treatment with salicylic acid decreases the effect of cadmium on photosynthesis in maize plants. - J. Plant Physiol. 165: 920-931, 2008

Liddycoat, S.M., Greenberg, B.M., Wolyn, D.J.: The effect of plant growth-promoting rhizobacteria on asparagus seedlings and germinating seeds subjected to water stress under greenhouse conditions. - Can. J. Microbiol. 55: 388-394, 2009.

Lowry, O.H., Rosebrough, N.J., Farr, A.L., Randall, R.J.: Protein measurement with the Folin phenol reagent. - J. biol. Chem. 193: 265-275, 1951.

Lu, Y., Li, Y., Zhang, J., Xiao, Y., Yue, Y., Duan, L., Zhang, M., Li, Z.: Overexpression of Arabidopsis molybdenum cofactor sulfurase gene confers drought tolerance in maize (Zea mays L.). - Plos ONE 8: e52126., 2013.

Lyons, R., Manners, J.M., Kazan, K.: Jasmonate biosynthesis and signaling in monocots: a comparative overview. - Plant Cell Rep. 32: 815-827, 2013.

Manivannan, P., Jaleel, C.A., Sankar, B., Kishorekumar, A., Somasundaram, R., Lakshmanan, G.A., Panneerselvam, R.: Growth, biochemical modifications and proline metabolism in Helianthus annuus L. as induced by drought stress. - Colloids Surf. B. Biointerfaces 59: 141-149, 2007.

Markwell, J.: The Minolta SPAD-502 leaf chlorophyll meter: an exciting new tool for education in the plant sciences. - Amer. Biol. Teaching 61: 672-676, 1999.

Marulanda, A., Barea, J.-M., Azcón, R.: Stimulation of plant growth and drought tolerance by native microorganisms (AM fungi and bacteria) from dry environments: mechanisms related to bacterial effectiveness. - J. Plant Growth Regul. 28: 115-124, 2009.

Marulanda, A., Barea, J., Azcon, R.: An indigenous droughttolerant strain of Glomus intraradices associated with a native bacterium improves water transport and root development in Retama sphaerocarpa. - Microbiol. Ecol. 52: 670-678, 2006.

Mittler, R.: Oxidative stress, antioxidants and stress tolerance. Trends Plant Sci. 7: 405-410, 2002.

Mohammadkhani, N., Heidari, R.: Effects of drought stress on soluble proteins in two maize varieties. - Turkish J. Biol. 32: 23-30, 2008.

Molinari, H.B.C., Marur, C.J., Daros, E., De Campos, M.K.F., De Carvalho, J.F.R.P., Pereira, L.F.P., Vieira, L.G.E.: Evaluation of the stress-inducible production of proline in transgenic sugarcane (Saccharum spp.): osmotic adjustment, chlorophyll fluorescence and oxidative stress. - Physiol. Plant. 130: 218229, 2007.

Moran, J.F., Becana, M., Iturbe-Ormaetxe, I., Frechilla, S., Klucas, R.V., Aparicio-Tejo, P.: Drought induces oxidative stress in pea plants. - Planta 194: 346-352, 1994.

Munns, R., Tester, M.: Mechanisms of salinity tolerance. - Annu. Rev. Plant Biol. 59: 651-681, 2008.

Nafie, E., Hathout, T., Mokadem, A., Shyma, A.: Jasmonic acid elicits oxidative defense and detoxification systems in
Cucumis melo L. cells. - Braz. J. Plant Physiol. 23: 161-174, 2011.

Nautiyal, C.S., Srivastava, S., Chauhan, P.S., Seem, K., Mishra, A., Sopory, S.K.: Plant growth-promoting bacteria Bacillus amyloliquefaciens NBRISN13 modulates gene expression profile of leaf and rhizosphere community in rice during salt stress. - Plant Physiol. Biochem. 66: 1-9, 2013.

Nayyar, H., Gupta, D.: Differential sensitivity of C3 and C4 plants to water deficit stress: association with oxidative stress and antioxidants. - Environ. exp. Bot. 58: 106-113, 2006.

Nogueirol, R.C., Monteiro, F.A., Gratão, P.L., Borgo, L., Azevedo, R.A.: Tropical soils with high aluminum concentrations cause oxidative stress in two tomato genotypes. - Environ. Monitor. Assess. 187: 1-16, 2015.

Parchmann, S., Gundlach, H., Mueller, M.J.: Induction of 12-oxophytodienoic acid in wounded plants and elicited plant cell cultures. - Plant Physiol. 115: 1057-1064, 1997.

Petrasek, J., Mravec, J., Bouchard, R., Blakeslee, J.J., Abas, M., Seifertova, D., Wisniewska, J., Tadele, Z., Kubes, M., Covanova, M., Dhonukshe, P., Skupa, P., Benkova, E., Perry, L., Krecek, P., Lee, O.R., Fink, G.R., Geisler, M., Murphy, A.S., Luschnig, C., Zazimalova, E., Friml, J.: PIN proteins perform a rate-limiting function in cellular auxin efflux. Science 312: 914-918, 2006.

Ray, S., Alves, P.C., Ahmad, I., Gaffoor, I., Acevedo, F.E., Peiffer, M., Jin, S., Han, Y., Shakeel, S., Felton, G.W., Luthe, D.S.: Turnabout is fair play: herbivory-induced plant chitinases excreted in fall armyworm frass suppress herbivore defenses in maize. - Plant Physiol. 171: 694-706, 2016.

Remy, E., Cabrito, T.R., Baster, P., Batista, R.A., Teixeira, M.C., Friml, J., Sa-Correia, I., Duque, P.: A major facilitator superfamily transporter plays a dual role in polar auxin transport and drought stress tolerance in Arabidopsis. - Plant Cell 25: 901-926, 2013.

Sarma, R.K., Saikia, R.: Alleviation of drought stress in mung bean by strain Pseudomonas aeruginosa GGRJ21. - Plant Soil 377: 111-126, 2014.

Shinozaki, K., Yamaguchi-Shinozaki, K.: Gene networks involved in drought stress response and tolerance. - J. exp. Bot. 58: 221-227, 2007.

Stürzenbaum, S.R., Kille, P.: Control genes in quantitative molecular biological techniques: the variability of invariance. - Comp. Biochem. Physiol. 130: 281-289, 2001.

Teulat, B., Zoumarou-Wallis, N., Rotter, B., Salem, M.B., Bahri, H., This, D.: QTL for relative water content in field-grown barley and their stability across Mediterranean environments. - Theor. appl. Genet. 108: 181-188, 2003.

Thudi, M., Upadhyaya, H.D., Rathore, A., Gaur, P.M., Krishnamurthy, L., Roorkiwal, M., Nayak, S.N., Chaturvedi, S.K., Basu, P.S., Gangarao, N.: Genetic dissection of drought and heat tolerance in chickpea through genome-wide and candidate gene-based association mapping approaches. - Plos ONE 9: e96758, 2014.

Vellosillo, T., Martínez, M., López, M.A., Vicente, J., Cascón, T., Dolan, L., Hamberg, M., Castresana, C.: Oxylipins produced by the 9-lipoxygenase pathway in Arabidopsis regulate lateral root development and defense responses through a specific signaling cascade. - Plant Cell. 19: 831-846, 2007.

Vinocur, B., Altman, A.: Recent advances in engineering plant tolerance to abiotic stress: achievements and limitations. Curr. Opin. Biotechnol. 16: 123-132, 2005.

Wahid, A., Gelani, S., Ashraf, M., Foolad, M.R.: Heat tolerance in plants: an overview. - Environ. exp. Bot. 61: 199-223, 2007.

Williams, M., Salas, J.J., Sanchez, J., Harwood, J.L.: Lipoxygenase pathway in olive callus cultures (Olea europaea). - Phytochemistry 53: 13-19, 2000. 
Xin, Z.Y., Zhou, X., Pilet, P.E.: Level changes of jasmonic, abscisic, and indole-3yl-acetic acids in maize under desiccation stress. - J. Plant Physiol. 151: 120-124, 1997.

Yang, X.Y., Jiang, W.J., Yu, H.J.: The expression profiling of the lipoxygenase $(L O X)$ family genes during fruit development, abiotic stress and hormonal treatments in cucumber (Cucumis sativus L.). - Int. J. mol. Sci. 13: 2481-2500, 2012.

Ye, Z., Rodriguez, R., Tran, A., Hoang, H., De los Santos, D., Brown, S., Vellanoweth, R.L.: The developmental transition to flowering represses ascorbate peroxidase activity and induces enzymatic lipid peroxidation in leaf tissue in Arabidopsis thaliana. - Plant Sci. 158: 115-127, 2000.

Zakikhani, H., Ardakani, M.R., Rejali, F., Gholamhoseini, M., Joghan, A.K., Dolatabadian, A.: Influence of diazotrophic bacteria on antioxidant enzymes and some biochemical characteristics of soybean subjected to water stress. - J. integr. Agr. 11: 1828-1835, 2012. 